Case Report

\title{
Insights into the Mechanism of Severe Mitral Regurgitation: RT-3D TEE Guided Management with Pathological Correlation
}

\author{
Senthil Anand, ${ }^{1}$ Naktal Hamoud, ${ }^{2}$ Jess Thompson, ${ }^{2}$ and Rajesh Janardhanan ${ }^{2}$ \\ ${ }^{1}$ Department of Medicine, Banner-University Medical Center South, Tucson, AZ 85713, USA \\ ${ }^{2}$ Department of Cardiovascular Medicine, Sarver Heart Center, Banner-University Medical Center, Tucson, AZ 85724, USA \\ Correspondence should be addressed to Senthil Anand; dr.anand.senthil@gmail.com
}

Received 25 June 2015; Accepted 28 October 2015

Academic Editor: Jesus Peteiro

Copyright (c) 2015 Senthil Anand et al. This is an open access article distributed under the Creative Commons Attribution License, which permits unrestricted use, distribution, and reproduction in any medium, provided the original work is properly cited.

\begin{abstract}
Mitral valve perforation is an uncommon but important complication of infective endocarditis. We report a case of a 65-year-old man who was diagnosed to have infective endocarditis of his mitral valve. Through the course of his admission he had a rapid development of hemodynamic instability and pulmonary edema secondary to acutely worsening mitral regurgitation. While the TEE demonstrated an increase in the size of his bacterial vegetation, Real Time 3D TEE was ultimately the imaging modality through which the valve perforation was identified. Through this case report we discuss the advantages that RT-3D TEE has over traditional 2D TEE in the management of valve perforation.
\end{abstract}

\section{Case Presentation}

A 65-year-old man was admitted with severe sepsis and bacteremia. He endorsed malaise, myalgia, fever, chills, and reduced appetite. His blood cultures were positive for Methicillin-sensitive Staphylococcus aureus. On admission, he was found to have acute renal failure and multiple septic brain emboli. The initial transthoracic echocardiogram (TTE) showed mild mitral regurgitation (MR) with no vegetation. In the presence of positive blood cultures and recurrent septic emboli, a 2D-transesophageal echocardiogram (TEE) was done on day 4 that revealed a $1.3 \mathrm{~cm} \times 0.3 \mathrm{~cm}$ wide mobile mass on the atrial side of the posterior mitral leaflet, suggestive of vegetation. While the patient was getting appropriate antibiotics, on day 12 , he developed an acute pulmonary edema and new onset of atrial fibrillation with rapid ventricular rate. $2 \mathrm{D}$-transesophageal echo revealed a much larger vegetation (Figure 1(a)) measuring $2.3 \mathrm{~cm}$ on the P2 scallop of the posterior leaflet of the mitral valve and severe MR. Real Time 3D Transesophageal Echocardiography (RT3D TEE) confirmed a large vegetation on the P2 scallop of the posterior mitral leaflet (Figure 1(b)). Color 3D Doppler revealed two mitral regurgitation jets in the left atrium, one jet with a central origin and one jet that was lateral (Figure 2), and we were able to easily identify a perforation in the
P2 scallop of the posterior mitral leaflet with an estimated area of $0.8 \mathrm{~cm}$. The surgery team was consulted and on day 25 the patient underwent a repair of the mitral valve with placement of a $25 \mathrm{~mm}$ Simulus flexible annuloplasty band. Intraoperatively, the perforation size and location on the P2 scallop of the posterior mitral valve (Figure 3(a)) exactly matched the preoperative 3D TEE findings (Figure 3(b)).

\section{Discussion}

Despite appropriate medical management of our patient's infective endocarditis there was a rapid development of hemodynamic instability resulting in pulmonary edema. This raised the suspicion that the patient had developed a structural valvular complication secondary to the infective endocarditis. RT-3D TEE confirmed valve leaflet perforation and thus provided a mechanistic insight into the new onset severe MR. The ability to manipulate the images in a threedimensional environment also enabled us to better communicate the valve pathology to the surgical team and guide further treatment including valve repair.

Mitral valve perforation is an uncommon but important complication of bacterial endocarditis [1]. While TEE is much superior to TTE in the diagnosis of mitral leaflet perforation (sensitivity 95\%, specificity 98) [2], RT-3D TEE has been 


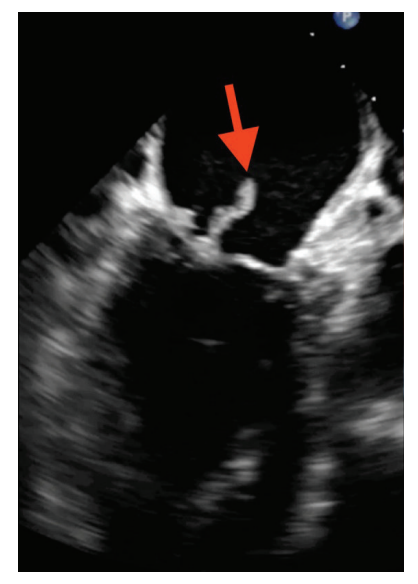

(a)

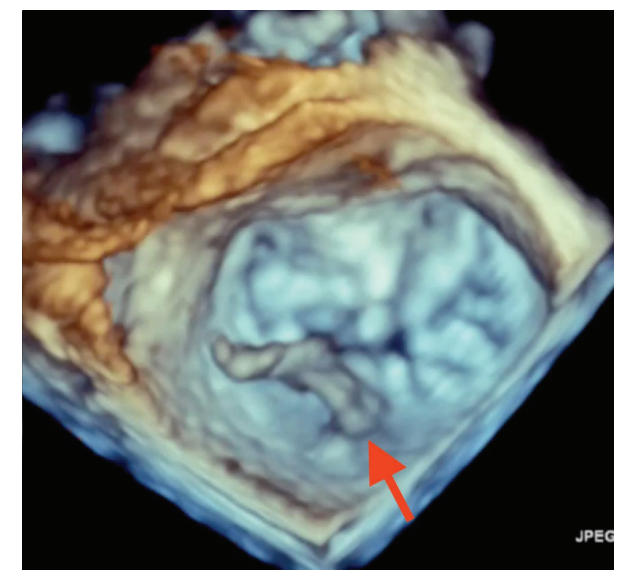

(b)

FIGURE 1: (a) shows the large vegetation on the P2 scallop of the posterior mitral valve on 2D TEE. (b) Corresponding mass noted on RT3DTEE.

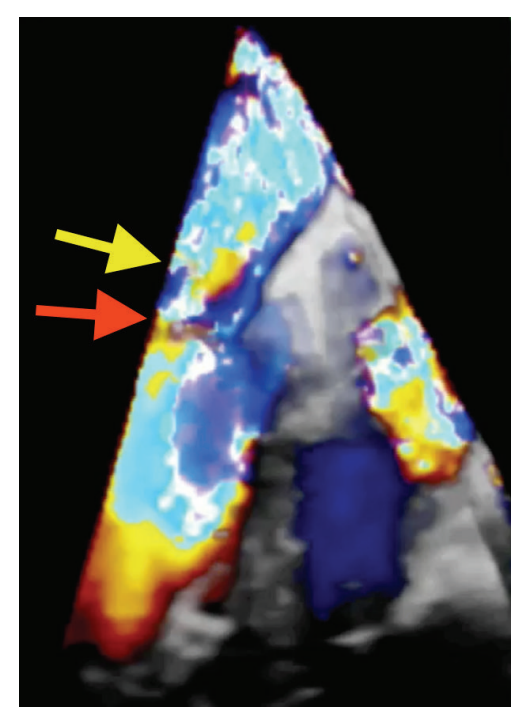

FIGURE 2: Color 3D TEE image on an Apical 4 Chamber view shows the impressive lateral jet (red arrow) originating from the perforation on the P2 scallop of posterior mitral leaflet. The less impressive central jet of mitral regurgitation is partially seen in this image (yellow arrow).
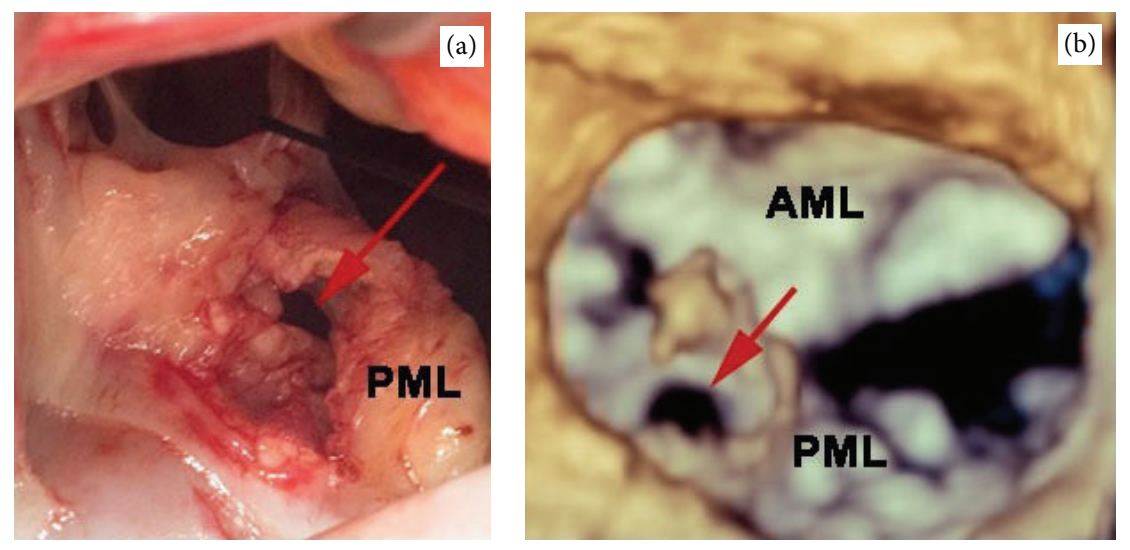

FIgURE 3: The red arrows on RT-3DTEE (a) and intraoperative image of the mitral valve (b) showing the perforation in the P2 scallop of the posterior mitral leaflet (AML: anterior mitral leaflet; PML: posterior mitral leaflet). 
shown to provide additional qualitative information on the mitral valve apparatus when compared with 2D TEE [3]. With its ability to attain en face anatomic views of the cardiac valves, RT-3D TEE has been shown to have higher sensitivity at detecting valve perforations, as well as better ability to characterize their size and location when compared with traditional 2D TEE [4]. RT-3D TEE provides optimal identification of the morphology and pathology of the mitral valve apparatus [5], and in endocarditis it has been reported to have high sensitivity and specificity [6].

In conclusion, this case demonstrates the important diagnostic value of RT-3DTEE for mitral valve perforation. Without any additional risk to the patients, the use of RT3D can improve the diagnostic power of TEE for valvular perforation; also, it can clearly identify the site of the perforation with accurate estimate of the size of the perforation and provides surgeons with relevant details to plan valve repair. Excellent surgical results have been reported after 3D TEE in such patients. We suggest that $3 \mathrm{D}$ techniques should be part of the standard TEE in all patients with endocarditis to ensure high diagnostic accuracy and anatomical information.

\section{Conflict of Interests}

The authors declare that there is no conflict of interests regarding the publication of this paper.

\section{References}

[1] S. A. Schwalm, L. Sugeng, J. Raman, V. Jeevanandum, and R. M. Lang, "Assessment of mitral valve leaflet perforation as a result of infective endocarditis by 3-dimensional realtime echocardiography," Journal of the American Society of Echocardiography, vol. 17, no. 8, pp. 919-922, 2004.

[2] D. G. Cziner, B. P. Rosenzweig, E. S. Katz, A. M. Keller, W. G. Daniel, and I. Kronzon, "Transesophageal versus transthoracic echocardiography for diagnosing mitral valve perforation," The American Journal of Cardiology, vol. 69, no. 17, pp. 1495-1497, 1992.

[3] A. Salustri, A. E. Becker, L. Van Herwerden, W. B. Vletter, F. J. Ten Cate, and J. R. T. C. Roelandt, "Three-dimensional echocardiography of normal and pathologic mitral valve: a comparison with two-dimensional transesophageal echocardiography," Journal of the American College of Cardiology, vol. 27, no. 6, pp. 1502-1510, 1996.

[4] K. A. Thompson, T. Shiota, K. Tolstrup, S. V. Gurudevan, and R. J. Siegel, "Utility of three-dimensional transesophageal echocardiography in the diagnosis of valvular perforations," The American Journal of Cardiology, vol. 107, no. 1, pp. 100-102, 2011.

[5] E. E. Salcedo, R. A. Quaife, T. Seres, and J. D. Carroll, "A framework for systematic characterization of the mitral valve by real-time three-dimensional transesophageal echocardiography," Journal of the American Society of Echocardiography, vol. 22, no. 10, pp. 1087-1099, 2009.

[6] Y. Kanzaki, K. Yoshida, T. Hozumi et al., "Evaluation of mitral valve lesions in patients with infective endocarditis by threedimensional echocardiography," Journal of Cardiology, vol. 33, no. 1, pp. 7-11, 1999. 


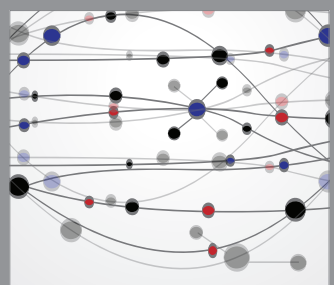

The Scientific World Journal
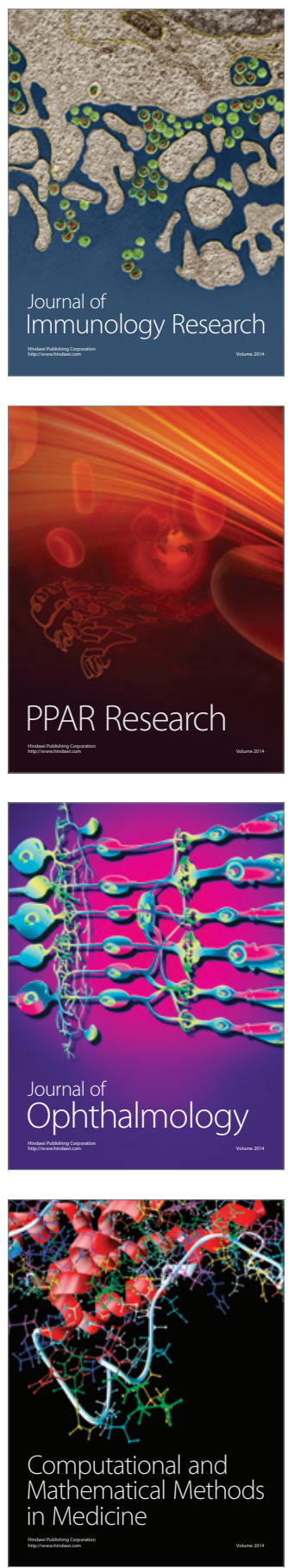

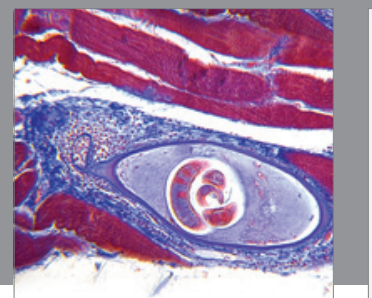

Gastroenterology

Research and Practice
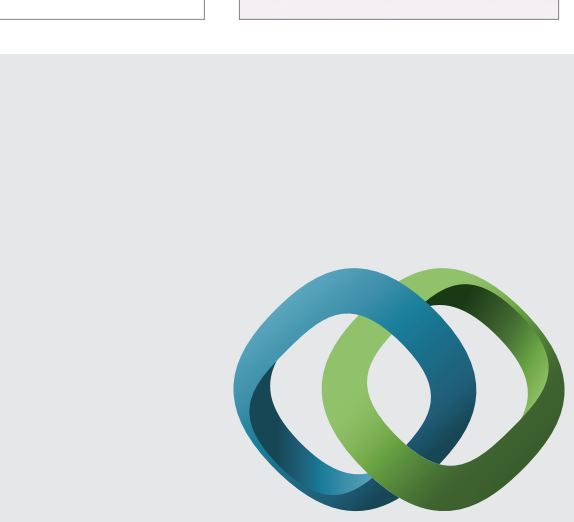

\section{Hindawi}

Submit your manuscripts at

http://www.hindawi.com
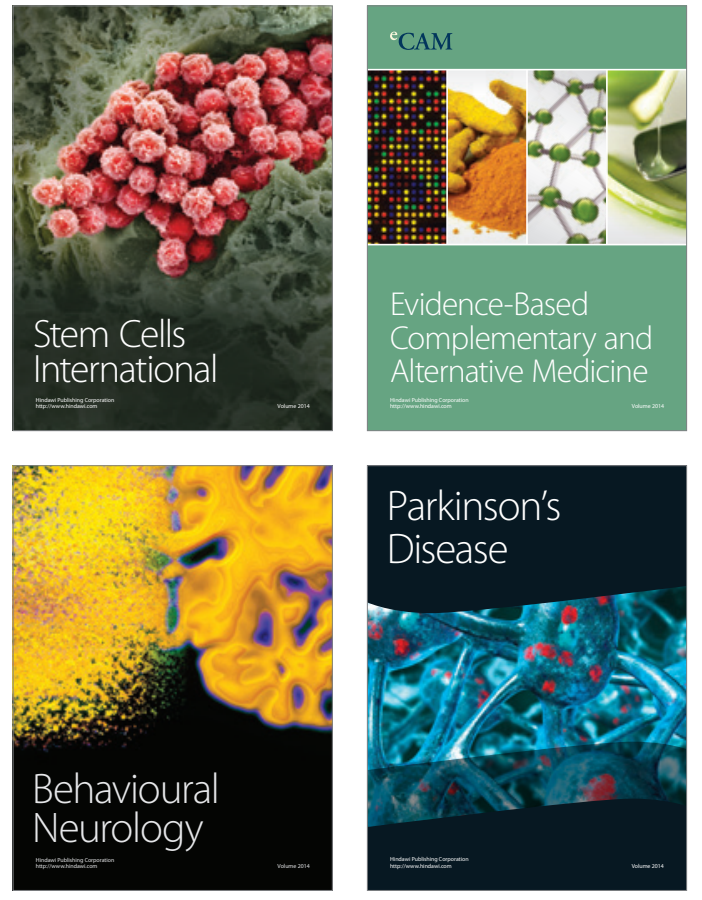
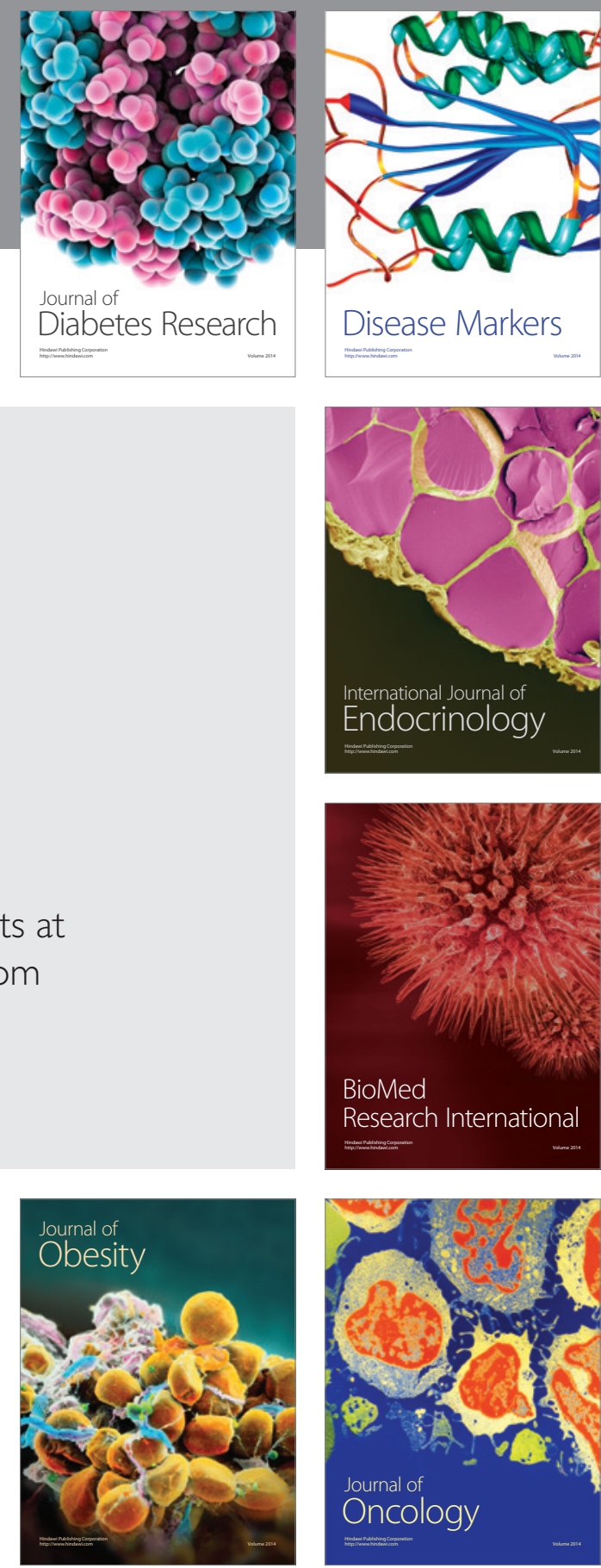

Disease Markers
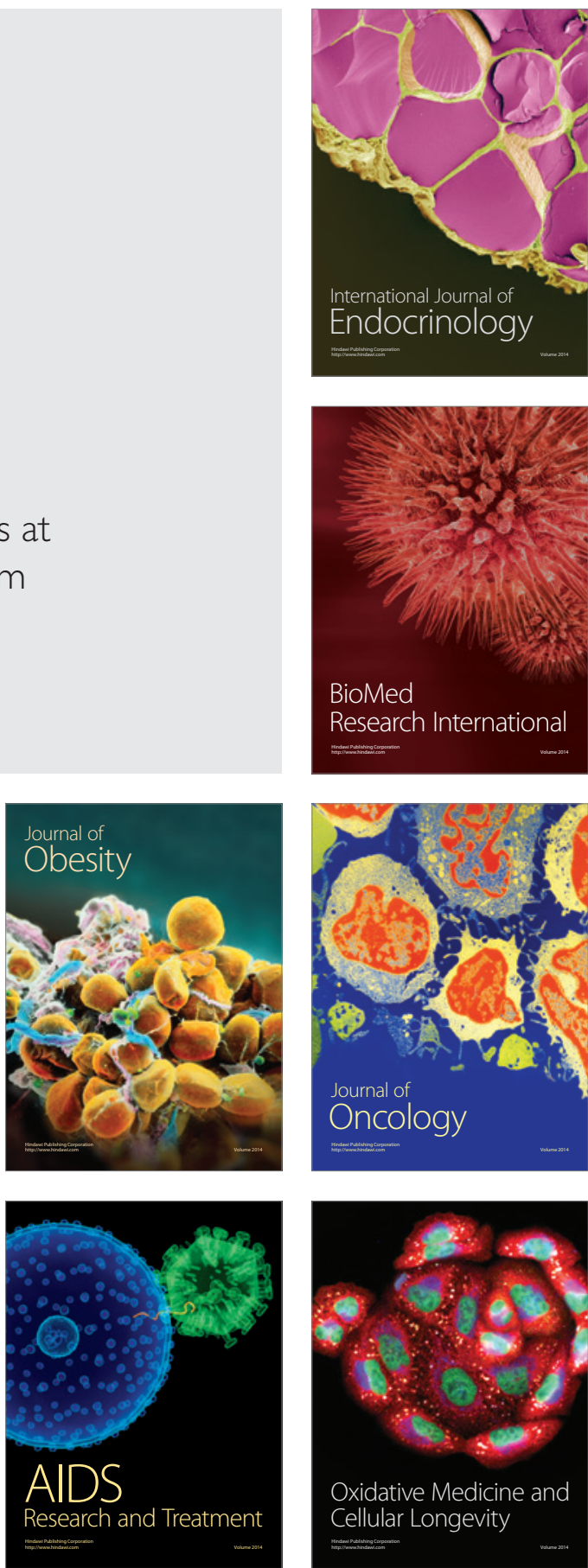